Patrick F. Cunniff

George J. O'Hara

University of Maryland College Park, MD 20742

\section{Feasibility of a Transient Dynamic Design Analysis Method}

This article addresses the degree of success that may be achieved by using simple equipment-vehicle models that produce time history responses whose equipment fixed base modal maximum response values are equivalent to those found in the U.S. Navy's dynamic design analysis method. The criteria of success is measured by a comparison of the results with typical interim shock design values. The equipment models reported are limited to two-degree and three degrees of freedom systems; the model of the vehicle to which the equipment is attached consists solely of a rigid mass and an elastic spring; and the shock excitation is produced by an ideal impulse that is applied to the vehicle mass. (C) 1994 John Wiley \& Sons, Inc.

\section{INTRODUCTION}

The dynamic design analysis method (DDAM) (Belsheim and O'Hara, 1960), has been used for more than 30 years as part of the Navy's efforts to shock-harden heavy shipboard equipment. This spectral method, which has been validated several times (O'Hara, 1979), employs normal mode theory, modal effective mass (O'Hara and Cunniff, 1963, Cunniff and O'Hara, 1965), and design shock values (O'Hara and Belsheim, 1963). DDAM prescribes a modal analysis approach that utilizes these shock design values in three orthogonal directions and takes into account the type of vehicle and equipment location, that is, hull-mounted, deck-mounted, and shell-plate mounted. Recent articles have provided an overview on the evolution of spectral techniques in naval shock design (Remmers, 1983), guidance to account for structural interactive effects in choosing design shock values from shock spectra (O'Hara and Cunniff, 1985), and the demonstration of a procedure that utilizes modal effective weight (Cunniff and O'Hara,
1989) for establishing shock design curves for spectral analysis from accumulated data.

Since its introduction, different transient analysis methods have been proposed as alternative approaches. The practice of using some "typical" base motion for a class of structural systems as a design input is flawed, sometimes grievously so. This is because during a shock, the base motion includes the interactive effects between the equipment and its supporting structure. Consequently, it is generally not useful as an input function for different equipment attached to the same supporting structure because the different interaction causes a different motion. Likewise, trying to average base motions obtained at different points of a multifoundation system can and does lead to a wide range of results that are in error. This is especially true when one compares shock design values derived from original base motions with those derived from average motions. Another method (Private communications, 1990), used a simple base mass to represent the vehicle to which the equipment is attached, and an impulsive force applied to the base mass to 
produce the shock motion excitation for the equipment. A recent article by O'Hara and Cunniff (1991) examined the degree of success that may be achieved by the simple model to produce time history base motions for the equipment responses whose modal maximum response values are the prescribed shock design values. The vehicle, modeled as a lumped mass, provided solutions when the ratio of the prescribed shock design values (mode 1 divided by mode 2) was less than unity. Solutions were unattainable when the same ratio of design inputs were equal to or greater than unity.

This study examines the situation when the vehicle model is a simple mass-spring system onto which a two or three degrees of freedom equipment is attached. The completed equipment-vehicle system is then excited by an impulse applied to the vehicle mass. The relative displacement of each equipment modal oscillator, scaled by its corresponding fixed base frequency, is compared to the shock design values. It will be shown that this new vehicle overcomes the shortcomings reported by O'Hara and Cunniff (1991) for two degrees of freedom equipment, and successfully accommodates the three degrees of freedom equipment.

\section{BACKGROUND}

Consider the equipment attached to the vehicle in Fig. 1(a) that is subject to a shock excitation. The equipment may be replaced by a dynamically equivalent modal model composed of its normal mode oscillators as shown in Fig. 1(b), where dynamical equivalence is defined as the response of the vehicle and the equipment-vehicle boundary being identical for the systems in Fig. 1(a,b). The mass of each oscillator is called the modal effective mass and the frequency of

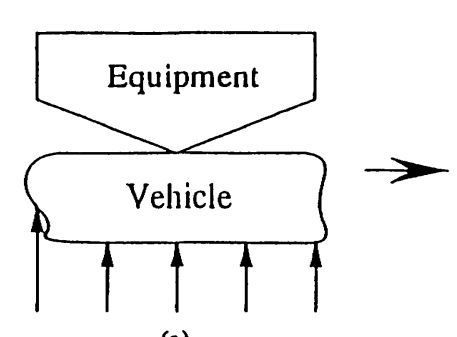

(a)

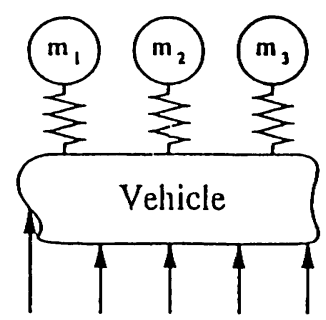

(b)
FIGURE 1 Equipment modeled by its modal oscillators.

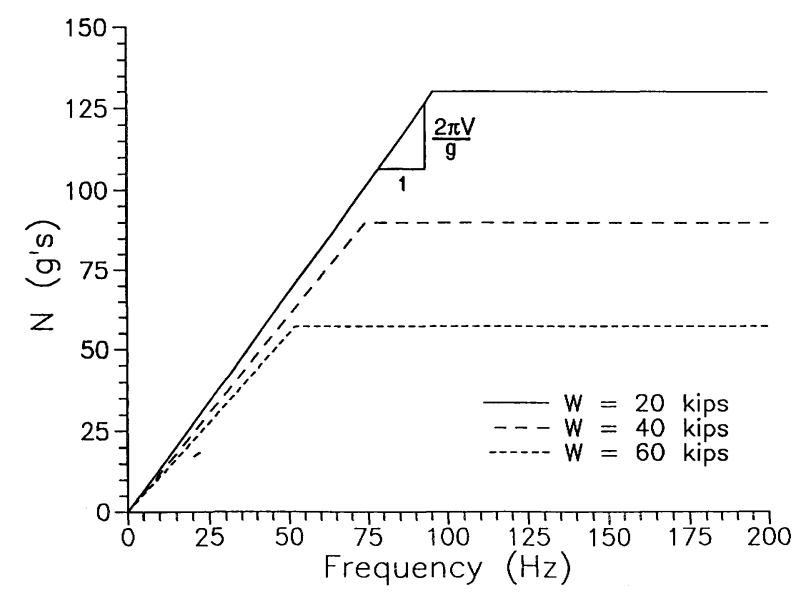

FIGURE 2 Typical shock design curves for different modal weights as a function of the fixed base frequencies.

each oscillator is selected to equal the corresponding fixed base natural frequency of the equipment. Knowing these modal characteristics, we can calculate the set of modal shock design values, $N$, from measured field data. Being cognizant of the interaction effects between the equipment and its supporting structure along with the shock spectrum dip effect (O'Hara and Cunniff, 1985), carefully selected shock design values can be summarized by design curves such as those in Fig. 2. A way of arriving at these curves can be found as reported by Cunniff and O'Hara (1989). Having the modal shock design values, the characteristic loads are applied to the equipment model in order to calculate the modal deflections and stresses. These final modal quantities are added by means of the NRL sum. This is essentially the DDAM approach for estimating the maximum deflections and stresses experienced by heavy equipment.

Each design curve in Fig. 2 is for a constant modal effective weight. Observe that as the equipment fixed base frequency increases, the shock design value increases until the frequency reaches the corner frequency $f_{c}$, after which the value $N$ remains constant. An alternative way of viewing the design inputs is to consider the two lines that intersect at $f_{c}$ as shown in Fig. 3(a). One chooses the smaller of the two values of $N$ at a frequency $f$ for a given modal effective weight. The linear portion of the design curve is given by

$$
N=\left(\omega^{2} \frac{X}{g}\right)=\left(2 \pi \frac{V_{a}}{g}\right) f
$$




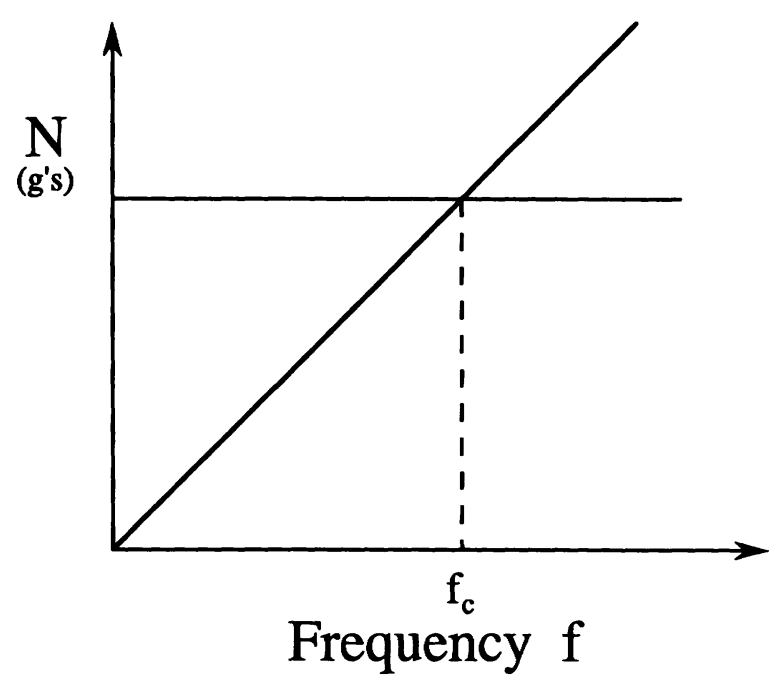

(a)

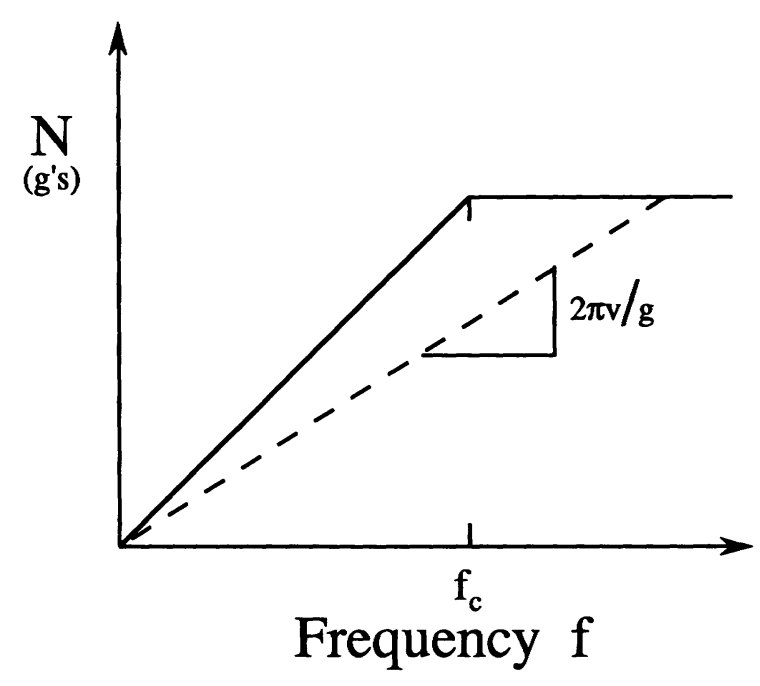

(b)

FIGURE 3 (a) The acceptable value of $N$ for a given frequency $f$ is the lesser of the two available values. (b) Example for finding the pseudo velocity $v$ when $f>f_{c}$.

in which $f$ is the fixed base frequency of the equipment in $\mathrm{Hz}$, and $V_{a}=\omega X$ is the maximum pseudovelocity. The maximum value of the acceleration $N$ for the given modal effective mass is also known, so that Eq. (1) is applicable up to the corner frequency $f_{c}$, where

$$
f_{c}=\left(N_{a} g\right) /\left(2 \pi V_{a}\right) .
$$

If the fixed base frequency of the equipment is greater than the corner frequency and the value of the equivalent pseudovelocity is desired at that frequency, then $V_{a}$ is reduced to $\nu$ as shown in Fig. 3(b) so that the modal acceleration remains $N_{a}$ at the frequency $f$.

In place of using design curves the following set of typical equations (O'Hara and Belsheim, 1963), are used here for calculating the pseudovelocity and shock design values, respectively, for a given modal effective weight $W_{a}$ in kips:

$$
V_{a}=20 \frac{\left(480+W_{a}\right)}{\left(100+W_{a}\right)} \quad \text { in./s) }
$$

and

$N_{a}=10.4 \frac{\left(480+W_{a}\right)}{\left(20+W_{a}\right)} \quad$ (number of g's).

Once again it is emphasized that the lesser acceleration shown in Fig. 3(a) is the shock design value used.

\section{TWO DEGREES OF FREEDOM EQUIPMENT}

\section{Problem Statement}

Figure 4 shows the vehicle consisting of mass $M_{0}$ and spring $K_{0}$ supporting two degrees of freedom equipment represented by its modal oscillators. $M_{1}$ and $M_{2}$ are the modal effective masses, $K_{1}$ and $K_{2}$ are the modal springs, and $\beta$ and $\gamma$ are the fixed base frequencies of the equipment, where $\beta$ $<\gamma$. The system is excited by an impulse applied to the base mass. It is assumed that the fixed base equipment modal characteristics are known so that the shock design values can be calculated in

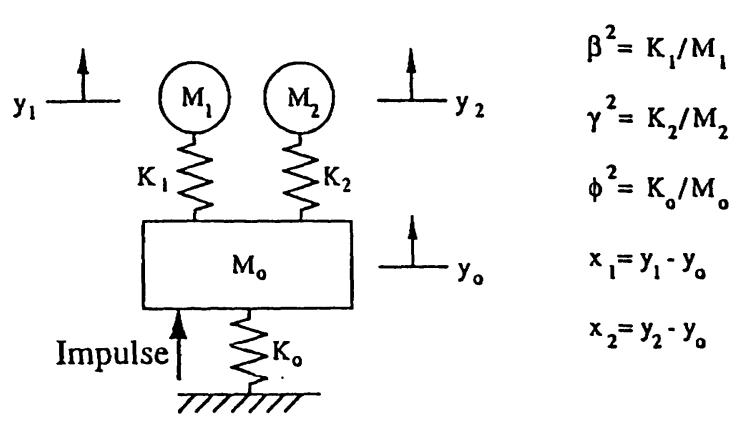

FIGURE 4 Two-degrees of freedom equipment attached to the vehicle $M_{0}, K_{0}$. 
the form of the pseudovelocities $\beta X_{1}$ and $\gamma X_{2}$ from Eqs. (3) and (4).

The problem centers on selecting the base mass, the vehicle frequency $\phi$, where $\phi^{2}=K_{0} /$ $M_{0}$, and the magnitude of the impulse velocity $V_{0}$ so that the ensuring time history motion of the modal model produces an equivalent shock damage potential. Thus, if we scale the modal oscillator relative displacement $X_{1}$ by $\beta$ and $X_{2}$ by $\gamma$, the peak amplitudes of each time history should be equal to the shock design values $\beta X_{1}$ and $\gamma X_{2}$ thus establishing equivalence.

\section{Analysis}

The frequency equation for the three degrees of freedom system in Fig. 4 is

$$
\begin{array}{r}
\left(\phi^{2}-\omega^{2}\right)\left(\beta^{2}-\omega^{2}\right)\left(\gamma^{2}-\omega^{2}\right)-\mu \beta^{2} \omega^{2}\left(\gamma^{2}-\omega^{2}\right) \\
-\tau \gamma^{2} \omega^{2}\left(\beta^{2}-\omega^{2}\right)=0
\end{array}
$$

in which $\mu=M_{1} / M_{0}$ and $\tau=M_{2} / M_{0}$. A general schematic locating the roots $\omega_{1}, \omega_{2}$, and $\omega_{3}$ is shown in Fig. 5. The region for the equipment fixed base frequency $\beta$, where $\omega_{1}<\beta<\omega_{2}$, and the fixed base frequency $\gamma$, where $\omega_{2}<\gamma<\omega_{3}$, are shown along with the vehicle frequency $\phi$.

The response of the equipment modal mass $M_{2}$ relative to the base mass is:

$$
\begin{aligned}
-X_{2} / V_{0}= & \left(A / \omega_{1}\right) \sin \omega_{1} t+\left(B / \omega_{2}\right) \sin \omega_{2} t \\
& +\left(C / \omega_{3}\right) \sin \omega_{3} t
\end{aligned}
$$

in which

$$
\begin{aligned}
& A=\left(\omega_{1}^{4}-\omega_{1}^{2} \beta^{2}\right) /\left(\omega_{2}^{2}-\omega_{1}^{2}\right)\left(\omega_{3}^{2}-\omega_{1}^{2}\right) \\
& B=\left(\omega_{2}^{4}-\omega_{2}^{2} \beta^{2}\right) /\left(\omega_{1}^{2}-\omega_{2}^{2}\right)\left(\omega_{3}^{2}-\omega_{2}^{2}\right) \\
& C=\left(\omega_{3}^{4}-\omega_{3}^{2} \beta^{2}\right) /\left(\omega_{1}^{2}-\omega_{3}^{2}\right)\left(\omega_{2}^{2}-\omega_{3}^{2}\right) .
\end{aligned}
$$

It can be shown that

$$
\left|X_{2} / V_{0}\right|_{\max }=Q / D
$$

in which

$$
\begin{aligned}
Q= & \left(\beta^{2} \omega_{1}^{2}-\omega_{1}^{4}\right)\left(\omega_{3}^{2}-\omega_{2}^{2}\right) \omega_{2} \omega_{3} \\
& +\left(\omega_{2}^{4}-\omega_{2}^{2} \beta^{2}\right)\left(\omega_{3}^{2}-\omega_{1}^{2}\right) \omega_{3} \omega_{1} \\
& +\left(\omega_{3}^{4}-\omega_{3}^{2} \beta^{2}\right)\left(\omega_{2}^{2}-\omega_{1}^{2}\right) \omega_{1} \omega_{2} \\
D= & \omega_{1} \omega_{2} \omega_{3}\left(\omega_{2}^{2}-\omega_{1}^{2}\right)\left(\omega_{3}^{2}-\omega_{1}^{2}\right)\left(\omega_{3}^{2}-\omega_{2}^{2}\right) .
\end{aligned}
$$

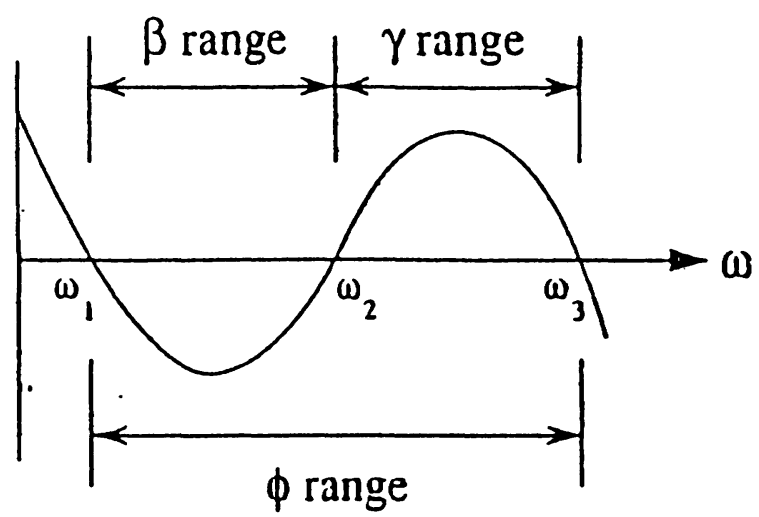

FIGURE 5 Range of the system frequencies, equipment fixed-base frequencies, and the vehicle frequency.

Likewise,

$$
\left|X_{1} / V_{0}\right|_{\max }=R / D
$$

in which

$$
\begin{aligned}
R= & \left(\gamma^{2} \omega_{1}^{2}-\omega_{1}^{4}\right)\left(\omega_{3}^{2}-\omega_{2}^{2}\right) \omega_{2} \omega_{3} \\
& +\left(\gamma^{2} \omega_{2}^{2}-\omega_{2}^{4}\right)\left(\omega_{3}^{2}-\omega_{1}^{2}\right) \omega_{1} \omega_{3} \\
& +\left(\omega_{3}^{4}-\omega_{3}^{2} \gamma^{2}\right)\left(\omega_{2}^{2}-\omega_{1}^{2}\right) \omega_{1} \omega_{2} .
\end{aligned}
$$

Equations (7) and (8) form the ratio of the shock design values as:

$$
r=\beta X_{1} / \gamma X_{2}=\beta R / \gamma Q .
$$

Knowing the ratio $r$ from Eqs. (2)-(4), the problem is to find $W_{0}$ and $\phi$ so that the terms on the right side of Eq. (9) are satisfied. Once this is achieved, Eqs. (7) and (8) may be rearranged to find the relationship for $V_{0}$ as:

$$
V_{0}=\beta X_{1} D / \beta R \text { or } \quad V_{0}=\gamma X_{2} D / \gamma Q .
$$

The transient modal model is now complete. Assigning the initial velocity $V_{0}$ to the base of weight $W_{0}$ and knowing the vehicle frequency $\phi$, the ensuing time history motion of $\beta X_{1}$ and $\gamma X_{2}$ should produce peak values equal to the prescribed shock design values, when each modal oscillator is treated as a fixed base system, and subject to the base motion of $W_{0}$.

It is interesting to let $W_{0}=\infty$ so that $\mu$ and $\tau$ are zero in Eq. (5). It follows that the system frequencies are $\phi, \beta$, and $\gamma$ Equation (9) reduces 
to

$$
r=\beta(\gamma-\phi) /[\gamma(\mathrm{B}-\phi)]
$$

from which $\phi$ is found:

$$
\phi=\beta \frac{(1-r)}{\left(\frac{\beta}{\gamma}-r\right)} .
$$

Because $\phi$ must be positive and $\beta<\gamma$, there is a gap where Eq. (12) holds, namely, $\beta / \gamma<r<1$. It was observed that for the limited number of examples studied to date, the values of $\phi$ less than $\phi$ in Eq. (12) could never yield a transient model that satisfies its DDAM design ratio $r$.

Finally, if we let $W_{0}$ approach zero in Eq. (9), it reduces to

$$
r=\beta / \gamma
$$

\section{Example 1}

Let the modal effective weights for the two degrees of freedom equipment in Fig. 4 be 26 and 60 kips and the corresponding fixed base frequencies equal 27 and $61 \mathrm{~Hz}$, respectively. The shock design values, obtained from Eqs. (2)-(4) are the pseudovelocities $\beta X_{1}=80.31746 \mathrm{in} . / \mathrm{s}$ and $\gamma X_{2}=$

\begin{tabular}{|c|c|c|c|c|c|}
\hline \multicolumn{3}{|c|}{$\begin{array}{c}\text { Reference } \beta X_{1}= \\
80.3175 \text { in. } / \mathrm{s}\end{array}$} & \multicolumn{3}{|c|}{$\begin{array}{c}\text { Reference } \gamma X_{2}=67.5000 \\
\text { in./s }\end{array}$} \\
\hline Design & $\begin{array}{c}\phi \\
(\mathrm{Hz})\end{array}$ & $\begin{array}{c}W_{0} \\
\text { (kips) }\end{array}$ & $\begin{array}{c}V_{0} \\
\text { (in./s) }\end{array}$ & $\left|\beta X_{1}\right|_{\max }$ & $\left|\gamma X_{2}\right|_{\max }$ \\
\hline \multirow[t]{2}{*}{1} & 27 & 79.44 & 67.9910 & 79.0696 & 66.1933 \\
\hline & & & 69.0641 & 80.3175 & 67.2380 \\
\hline \multirow[t]{2}{*}{2} & 13.5 & 251.40 & 60.1793 & 79.9947 & 67.4454 \\
\hline & & & 60.2280 & 80.0594 & 67.5000 \\
\hline
\end{tabular}
$67.50000 \mathrm{in} . / \mathrm{s})$ so that the ratio $r=1.1899$. Note
Table 1. Example 1

that this is the system for which a solution was previously unattainable when the model vehicle spring $K_{0}$ was not present (O'Hara and Cunniff, 1991). Figure 6 contains plots of the ratio $r$ as a function of the base weight $W_{0}$ for fixed values of the vehicle frequency $\phi$. These include $\phi=\beta$ and $\phi=0.5 \beta$, both of which provide a value $W_{0}$ by intersecting the straight line representing the ratio $r=1.1899 ; \phi=\gamma=2.259 \beta$ that does not intersect the $r$-line; and $\phi=\phi_{\min }=0.254 \beta$ that approaches the $r$-line asymptotically as $W_{0}$ approaches infinity. Table 1 summarizes the results for the two designs that provide viable transient models. The values listed in the fifth and sixth columns are the maximum absolute values of the responses of the modal masses for the initial velocity $V_{0}$. After the first trial run, $V_{0}$ was scaled to provide a more accurate value of the required peak values of $\beta X_{1}$ and $\gamma X_{2}$. Figure 7(a,b) shows

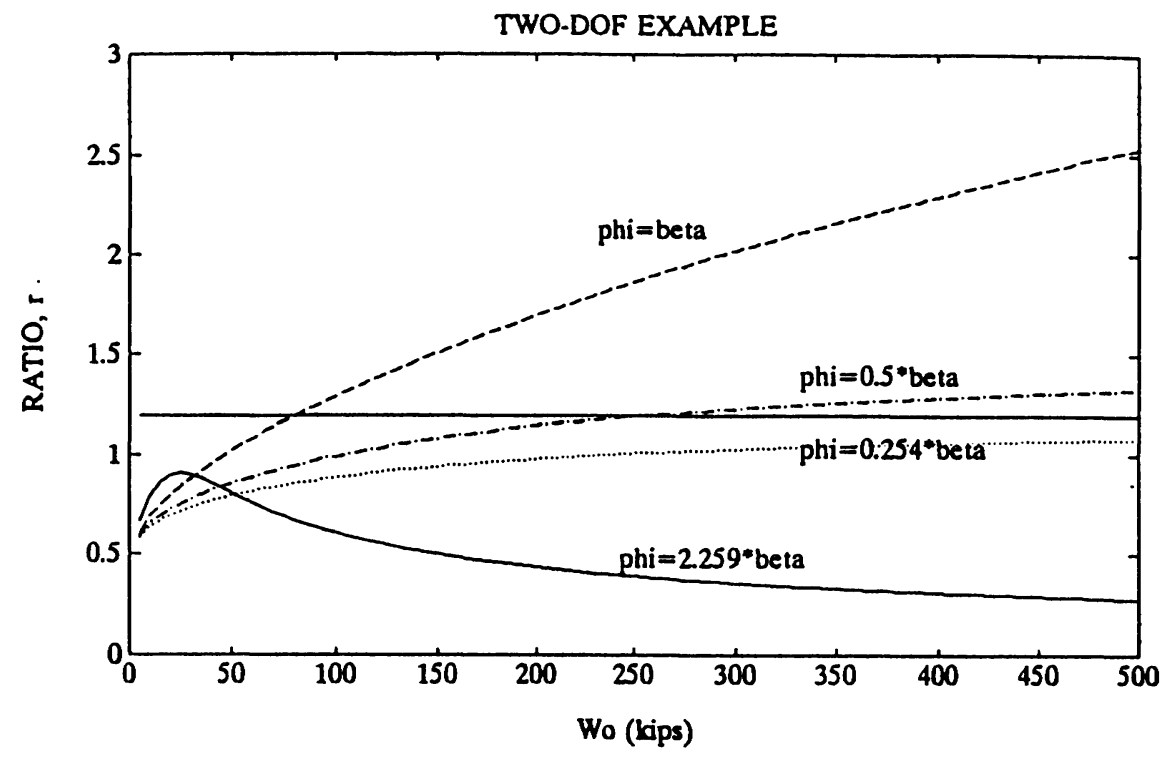

FIGURE 6 Shock design ratio as a function of the vehicle weight for fixed values of the vehicle frequency $\phi$. 
TWO-DOF EXAMPLE, DESIGN 1

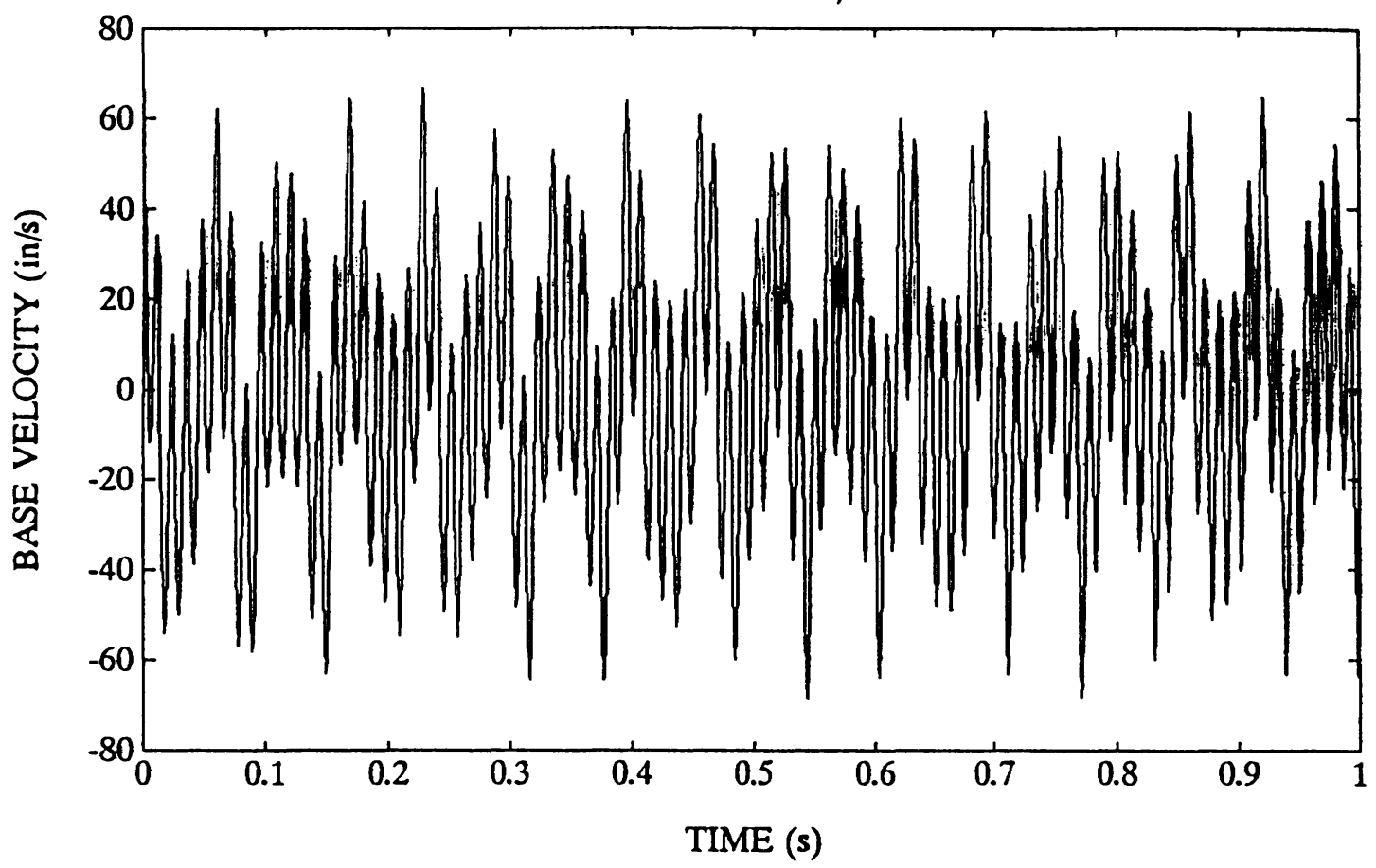

TWO-DOF EXAMPLE, DESIGN 2

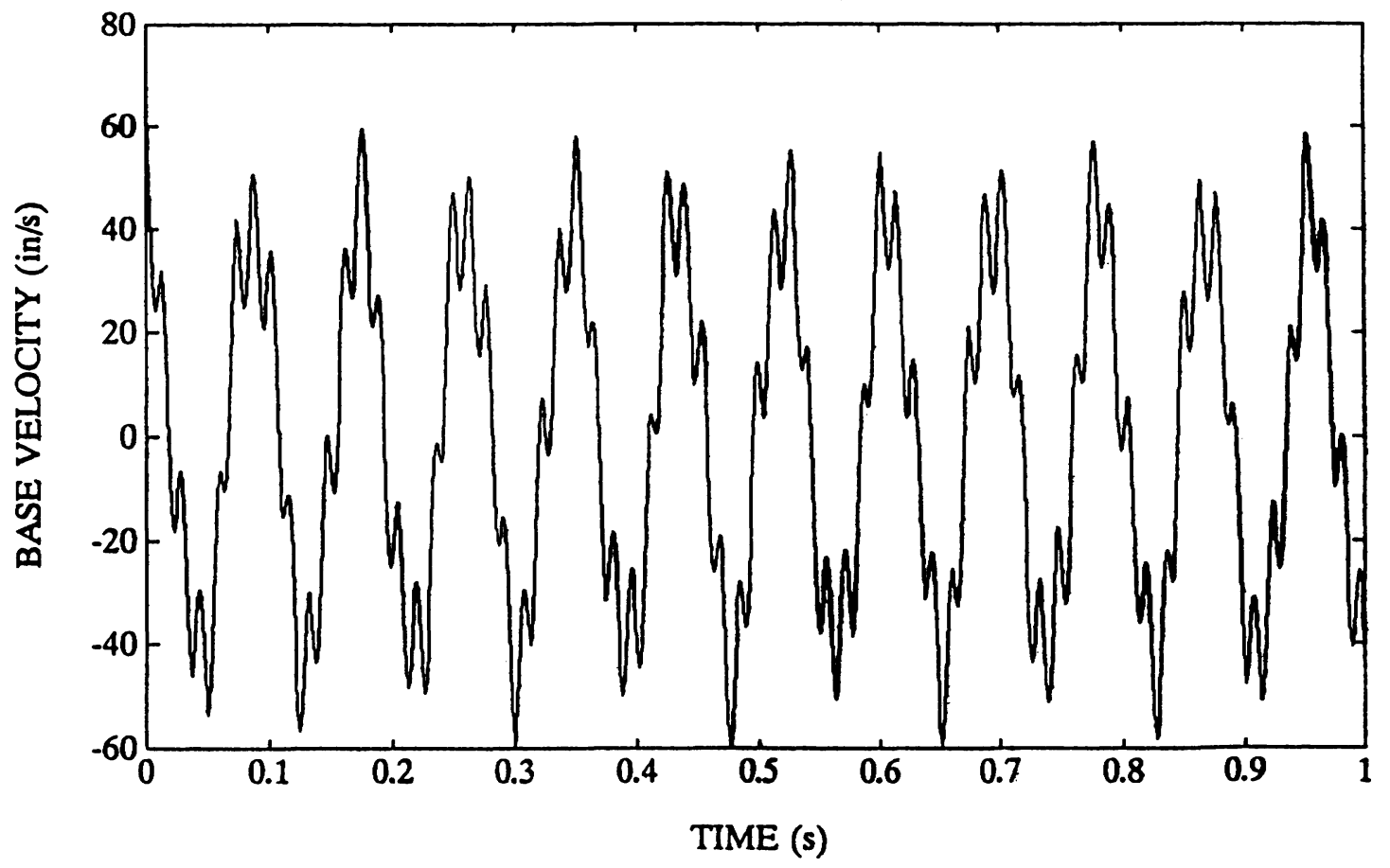

FIGURE 7 Time history motions, base velocity, Design 1 and Design 2, Example 1. 


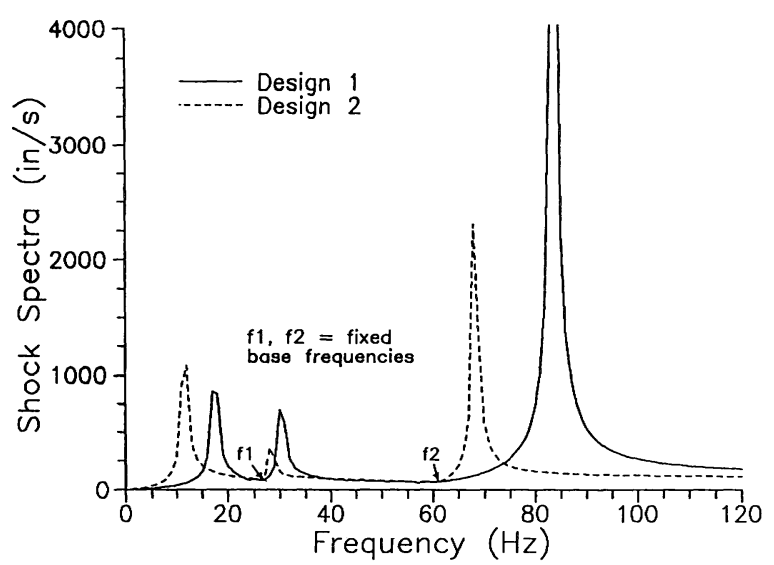

FIGURE 8 Shock response spectra for Designs 1 and 2.

the base velocity time history motions for each design. Figure 8 shows the shock response spectra generated from each of these vehicle motions. Note that because these base motions are different for the two designs, the corresponding shock response spectra are also different. However, we observe that the shock design values at the fixed base natural frequencies of the equipment are identical.

\section{THREE DEGREES OF FREEDOM EQUIPMENT}

Consider the modal model for the three degrees of freedom equipment shown in Fig. 9 attached to the vehicle of mass $M_{0}$ and spring $K_{0}$. As in the case of the two degrees of freedom equipment, the base mass is excited by an impulse producing an initial velocity $V_{0}$. Knowing the equipment fixed base frequencies $\beta, \gamma$, and $\delta$, where $\beta<\gamma<$ $\delta$, and the modal effective weights, the DDAMlike shock design values are calculated and labeled $\beta X_{1}, \gamma X_{2}$, and $\delta X_{3}$. The analysis of the maximum response of the relative motion of each modal oscillator is similar to the two degrees of freedom equipment except that there are now two independent ratios to satisfy from the three shock design ratios defined as follows:

$$
r_{1}=\beta X_{1} / \gamma X_{2} ; \quad r_{2}=\beta X_{1} / \delta X_{3} ; \quad r_{3}=\gamma X_{2} / \delta X_{3} .
$$

The equations for finding these ratios are summarized in Appendix A. It is emphasized that the desired vehicle parameters $W_{0}$ and $\phi$ must be found such that the two independent ratios are equal to their respective DDAM values. This re-

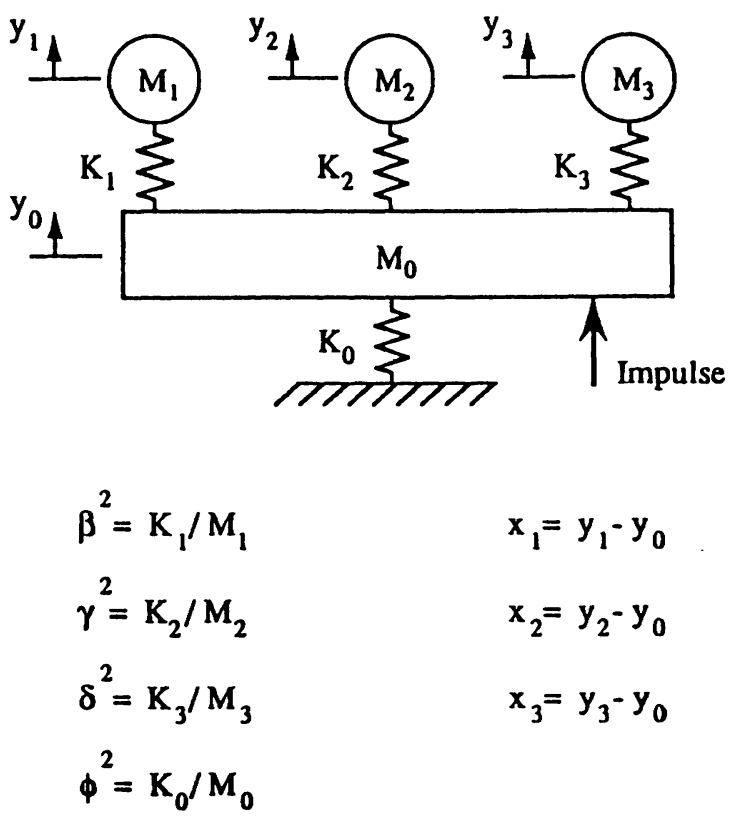

FIGURE 9 Three-degrees of freedom equipment attached to the vehicle $M_{0}, K_{0}$.

quires a trial and error process that is described in the following example.

\section{Example 2}

Table 2 lists the modal effective weights, the fixed base frequencies, and the shock design ratios for the three degrees of freedom modal equipment model in Fig. 9. A trial and error procedure is used to find the base frequency $\phi$ such that the plot of two of the ratios corresponding to the largest modal weights intersect their DDAM design ratio value at the same $W_{0}$. This is achieved by finding the value of $W_{0}$ at each point of intersection, say $W_{01}$ for the $r_{1}$ plot and $W_{02}$ for the $r_{2}$ plot. A solution is normally found rather rapidly by examining the change in sign in $\Delta W_{0}=$ $\left(W_{01}-W_{02}\right)$ as $\phi$ is varied. For this example a value of $\phi / \beta=0.9475$ was found after eight iterations that gave the results shown in Fig. 10. Note that all three $r$-plots intersect their design ratios at $W_{0}=455$ kips.

Table 2. Example 2

\begin{tabular}{cccc}
\hline$i$ & $\begin{array}{c}\text { Modal Weight, } W_{i} \\
\text { (kips) }\end{array}$ & $\begin{array}{c}\text { Frequency, } f_{i} \\
(\mathrm{~Hz})\end{array}$ & Ratio, $r_{i}$ \\
\hline 1 & 60 & 58 & 0.8406 \\
2 & 26 & 61 & 0.7576 \\
3 & 10 & 90 & 0.9012 \\
\hline
\end{tabular}




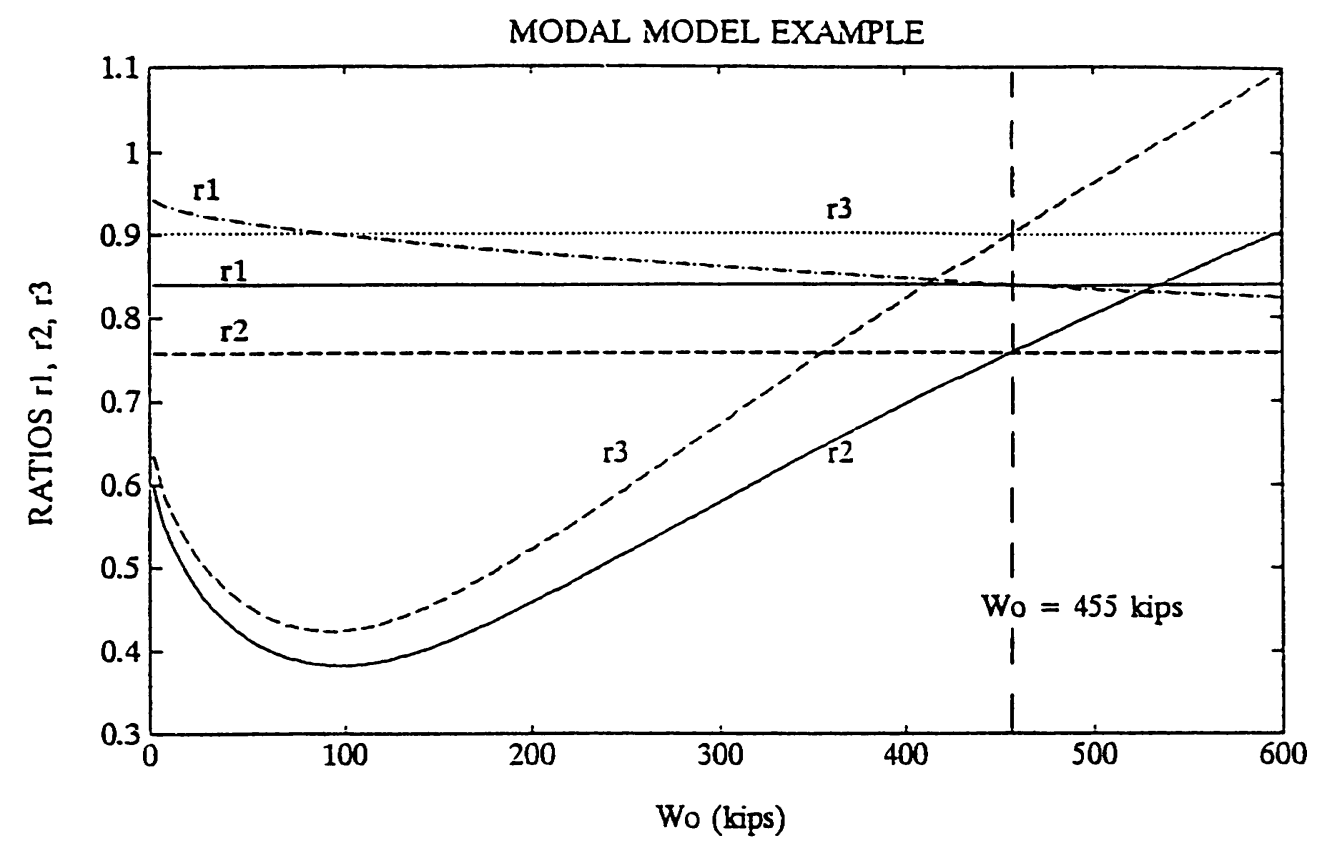

FIGURE 10 Shock design ratios versus the vehicle weight for $\phi=0.9475 \beta$.

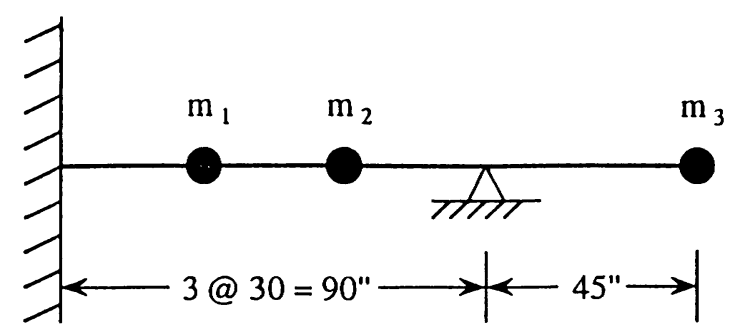

FIGURE 11 Example of an overhanging beam, $m_{1}=m_{2}=m_{3}$.

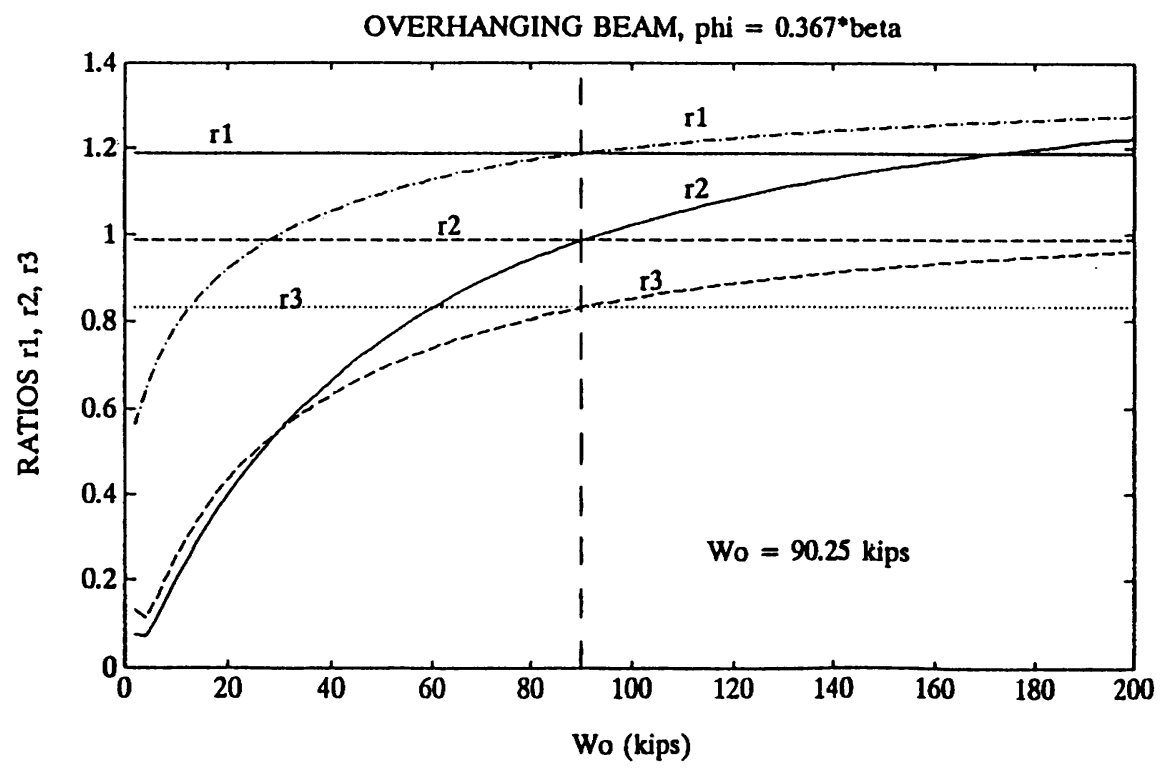

FIGURE 12 Shock design ratios versus the vehicle weight for $\phi=0.367 \beta$. 

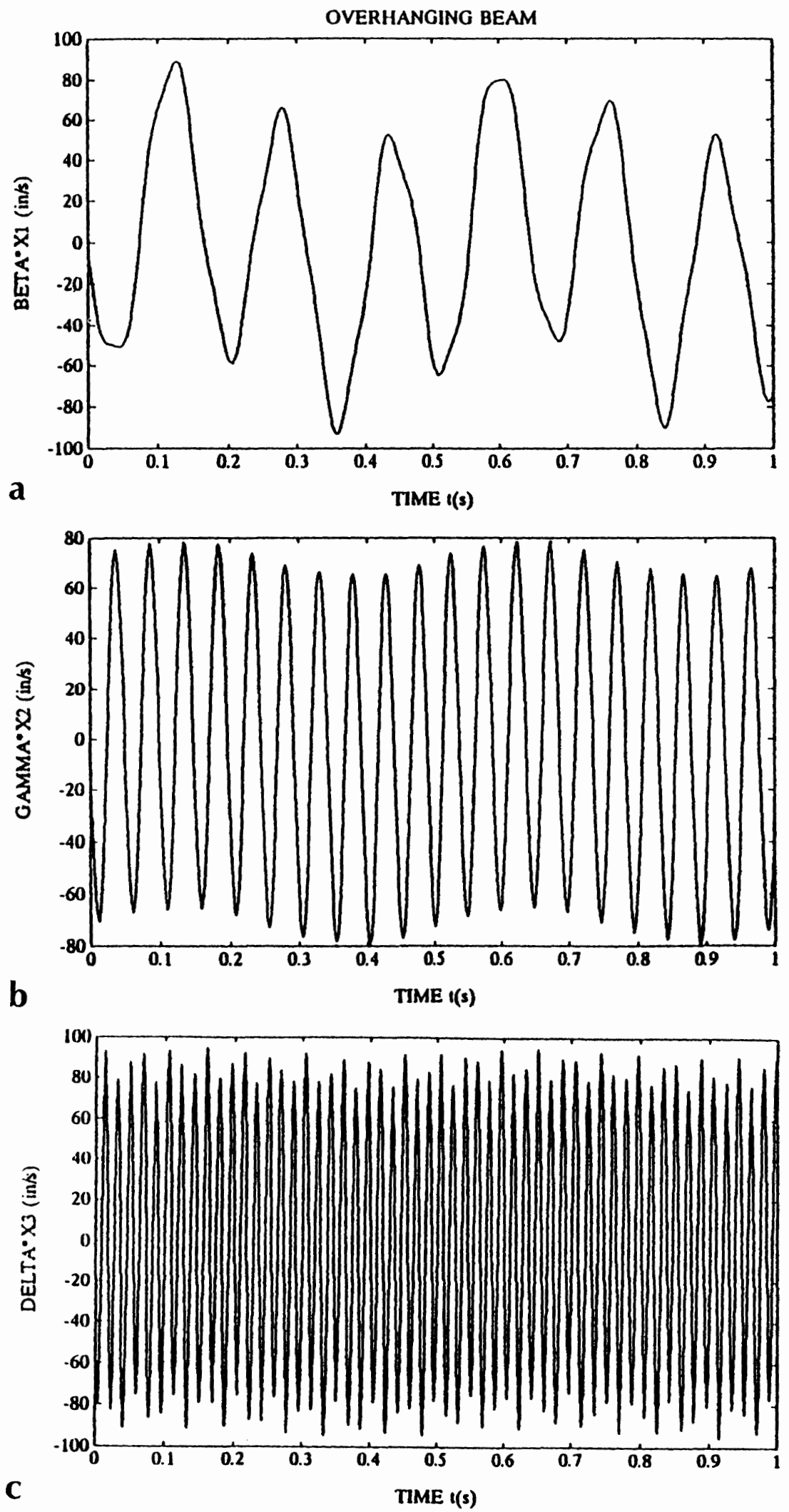

FIGURE 13 Time history motions for the overhanging beam; (a) $\beta X_{1}$, (b) $\gamma X_{2}$, (c) $\delta X_{3}$. 
Table 3. Example 3

\begin{tabular}{cccc}
\hline & $\begin{array}{c}\text { Modal Weight, } W_{i} \\
\text { (kips) }\end{array}$ & $\begin{array}{c}\text { Frequency, } f_{i} \\
(\mathrm{~Hz})\end{array}$ & Ratio, $r_{i}$ \\
\hline 1 & 2.103 & 6.205 & 1.1868 \\
2 & 27.572 & 17.884 & 0.9862 \\
3 & 0.325 & 54.808 & 0.8310 \\
\hline
\end{tabular}

\section{Example 3}

Let the overhanging beam support three equal lumped masses as shown in Fig. 11. Table 3 lists the modal effective weights, the fixed base frequencies, and the shock design ratios. Figure 12 shows the crossover points for each ratio occurring at $W_{0}=90.25$ kips for the case where $\phi=$ $0.367 \beta$. Figure $13(\mathrm{a}-\mathrm{c})$ shows the time responses of the scaled relative displacements where $V_{0}=$ $82.1748 \mathrm{in.} / \mathrm{s}$. Note that the system frequencies are present in these responses, where the system is composed of the beam's modal oscillators attached to the vehicle mass (Fig. 9). The measured peak values from these data points are compared with the DDAM shock design values as follows: $\beta X_{1}=93.013$ versus $94.435 \mathrm{in} . / \mathrm{s}$; $\gamma X_{2}=79.374$ versus $79.574 \mathrm{in} . / \mathrm{s} ; \delta X_{3}=95.754$ versus $95.754 \mathrm{in} . / \mathrm{s}$. The differences between the measured versus the desired values are due primarily to roundoff errors and a finite time increment of $0.001 \mathrm{~s}$ in the time history response.

\section{CONCLUSIONS}

The development of transient modal models that replicate the damaging potential prescribed by DDAM-like inputs has been described for two and three degrees of freedom equipment undergoing unidirectional motion. The vehicle is represented by a lumped mass and a grounded linear spring. The transient model is excited by an impulse applied to the vehicle mass. The two degrees of freedom equipment attached to such a vehicle model provides an infinite number of acceptable transient models. Example 1 clearly showed two acceptable transient models, each producing a different base motion, a different shock response spectra, but producing the same shock design values. Consequently, we conclude that there is no unique transient model because there are a multitude of transient models from which to choose.

The examples of the three degrees of freedom modal models presented herein showed only one transient model in each case. Further examination of the solutions may produce other acceptable transient models. This has been observed in a few instances and needs further exploration.

The next step in this study seeks to examine the more general case where the equipment has $n$ degrees of freedom, so that there are $(n-1)$ independent shock design ratios to satisfy. Attempts are underway to employ optimization techniques to expand the development of transient models that provide satisfactory replication of the DDAM-like inputs.

This study is part of a project that is supported by the Office of Naval Research. The content of this paper does not necessarily reflect the position or policy of the government, and no official endorsement should be inferred. Robert Wagner, a former mechanical engineering student, is acknowledged for his assistance with this project.

\section{REFERENCES}

Belsheim, R. O., and O'Hara, G. J., 1960, “Shock Design of Shipboard Equipment, Part I, DynamicDesign Analysis Method," NRL Report 5545.

Cunniff, P. F., and O'Hara, G. J., 1965, "Normal Mode Theory for Three-Dimensional Motion," NRL Report 6170.

Cunniff, P. F., and O'Hara, G. J., 1989, "'A Procedure of Generating Shock Design Values," Journal of Sound and Vibration, Vol. 134, pp. 155-164.

O'Hara, G. J., 1979, "'Shock Hardness Assessment of Submarine Equipment; Part V; Survival of Equipment Designed by DDAM," NRL Memorandum Report No. 3942, (U) Confidential Report, Unclassified Title.

O'Hara, G. J., and Belsheim, R. O., 1963, “'Interim Design Values for Shock Design of Shipboard Equipment,"' NRL Memorandum Report No. 1396.

O'Hara, G. J., and Cunniff, P. F., 1963, "Elements of Normal Mode Theory," NRL Report 6002.

O'Hara, G. J., and Cunniff, P. F., 1985, "The Shock Spectrum Dip Effect,' Journal of Sound Vibration, Vol. 103, pp. 311-321.

O'Hara, G. J., and Cunniff, P. F., 1991, “Time History Analysis of Systems as an Alternative to a DDAM-Type Analysis," Proceedings of the 62nd Shock \& Vibration Symposium, pp. 462-472.

Private communications among NAVSEA, TRC/ UERD, and the University of Maryland on a proposed transient design analysis method, Spring 1990.

Remmers, G., 1983, "Maurice Biot 50th Anniversary Lecture: The Evolution of Spectral Techniques in Navy Shock Design,' Shock and Vibration Bulletin, Part I, pp. 59-70. 


\section{APPENDIX A}

The following summarizes the equations for finding the maximum relative displacements of each of the three modal oscillators in Fig. 9:

$$
\left|\frac{x_{1}}{v_{0}}\right|_{\max }=\frac{n 1+n 2+n 3+n 4}{d}
$$

where

$$
\begin{gathered}
n 1=\omega_{1}\left(\gamma^{2}-\omega_{1}^{2}\right)\left(\delta^{2}-\omega_{1}^{2}\right)\left(\omega_{3}^{2}-\omega_{2}^{2}\right)\left(\omega_{4}^{2}-\omega_{2}^{2}\right)\left(\omega_{4}^{2}-\omega_{3}^{2}\right) \\
n 2=\omega_{2}\left(\gamma^{2}-\omega_{2}^{2}\right)\left(\delta^{2}-\omega_{2}^{2}\right)\left(\omega_{3}^{2}-\omega_{1}^{2}\right)\left(\omega_{4}^{2}-\omega_{1}^{2}\right)\left(\omega_{4}^{2}-\omega_{3}^{2}\right) \\
n 3=\omega_{3}\left(\omega_{3}^{2}-\gamma^{2}\right)\left(\delta^{2}-\omega_{3}^{2}\right)\left(\omega_{2}^{2}-\omega_{1}^{2}\right)\left(\omega_{4}^{2}-\omega_{1}^{2}\right)\left(\omega_{4}^{2}-\omega_{2}^{2}\right) \\
\left.n 4=\omega_{4}\left(\omega_{4}^{2}-\gamma^{2}\right)\left(\omega_{4}^{2}-\delta^{2}\right) \omega_{2}^{2}-\omega_{1}^{2}\right)\left(\omega_{3}^{2}-\omega_{1}^{2}\right)\left(\omega_{3}^{2}-\omega_{2}^{2}\right) \\
d=\left(\omega_{2}^{2}-\omega_{1}^{2}\right)\left(\omega_{3}^{2}-\omega_{1}^{2}\right)\left(\omega_{4}^{2}-\omega_{1}^{2}\right)\left(\omega_{3}^{2}-\omega_{2}^{2}\right)\left(\omega_{4}^{2}-\omega_{2}^{2}\right)\left(\omega_{4}^{2}-\omega_{3}^{2}\right) \\
\quad\left|\frac{x_{2}}{v_{0}}\right|_{\max }=\frac{n 5+n 6+n 7+n 8}{d}
\end{gathered}
$$

where

$$
\begin{aligned}
n 5 & =\omega_{1}\left(\beta^{2}-\omega_{1}^{2}\right)\left(\delta^{2}-\omega_{1}^{2}\right)\left(\omega_{3}^{2}-\omega_{2}^{2}\right)\left(\omega_{4}^{2}-\omega_{2}^{2}\right)\left(\omega_{4}^{2}-\omega_{3}^{2}\right) \\
n 6 & =\omega_{2}\left(\omega_{2}^{2}-\beta^{2}\right)\left(\delta^{2}-\omega_{2}^{2}\right)\left(\omega_{3}^{2}-\omega_{1}^{2}\right)\left(\omega_{4}^{2}-\omega_{1}^{2}\right)\left(\omega_{4}^{2}-\omega_{3}^{2}\right) \\
n 7 & =\omega_{3}\left(\omega_{3}^{2}-\beta^{2}\right)\left(\delta^{2}-\omega_{3}^{2}\right)\left(\omega_{2}^{2}-\omega_{1}^{2}\right)\left(\omega_{4}^{2}-\omega_{1}^{2}\right)\left(\omega_{4}^{2}-\omega_{2}^{2}\right) \\
n 8 & \left.\left.=\omega_{4}\left(\omega_{4}^{2}-\beta^{2}\right)\left(\omega_{4}^{2}-\delta^{2}\right)\left(\omega_{2}^{2}-\omega_{1}^{2}\right)\right)\left(\omega_{3}^{2}-\omega_{1}^{2}\right)\right)\left(\omega_{3}^{2}-\omega_{2}^{2}\right) \\
\left|\frac{x_{3}}{v_{0}}\right|_{\max } & =\frac{n 9+n 10+n 11+n 12}{d}
\end{aligned}
$$

where

$$
\begin{aligned}
n 9 & =\omega_{1}\left(\beta^{2}-\omega_{1}^{2}\right)\left(\gamma^{2}-\omega_{1}^{2}\right)\left(\omega_{3}^{2}-\omega_{2}^{2}\right)\left(\omega_{4}^{2}-\omega_{2}^{2}\right)\left(\omega_{4}^{2}-\omega_{3}^{2}\right) \\
n 10 & =\omega_{2}\left(\omega_{2}^{2}-\beta^{2}\right)\left(\gamma^{2}-\omega_{2}^{2}\right)\left(\omega_{3}^{2}-\omega_{1}^{2}\right)\left(\omega_{4}^{2}-\omega_{1}^{2}\right)\left(\omega_{4}^{2}-\omega_{3}^{2}\right) \\
n 11 & =\omega_{3}\left(\omega_{3}^{2}-\beta^{2}\right)\left(\omega_{3}^{2}-\gamma^{2}\right)\left(\omega_{2}^{2}-\omega_{1}^{2}\right)\left(\omega_{4}^{2}-\omega_{1}^{2}\right)\left(\omega_{4}^{2}-\omega_{2}^{2}\right) \\
n 12 & =\omega_{4}\left(\omega_{4}^{2}-\beta^{2}\right)\left(\omega_{4}^{2}-\gamma^{2}\right)\left(\omega_{2}^{2}-\omega_{1}^{2}\right)\left(\omega_{3}^{2}-\omega_{1}^{2}\right)\left(\omega_{3}^{2}-\omega_{2}^{2}\right) .
\end{aligned}
$$



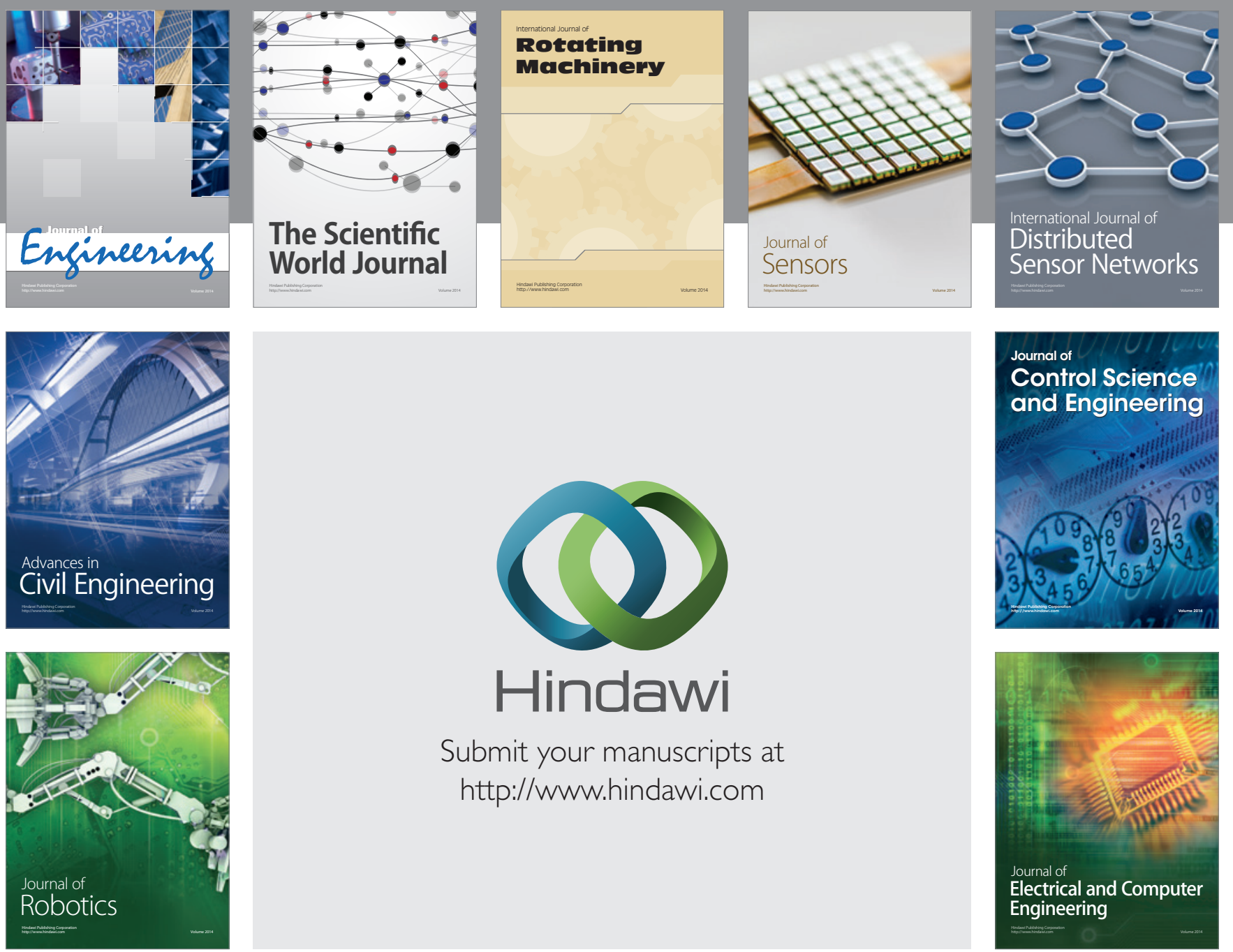

Submit your manuscripts at

http://www.hindawi.com
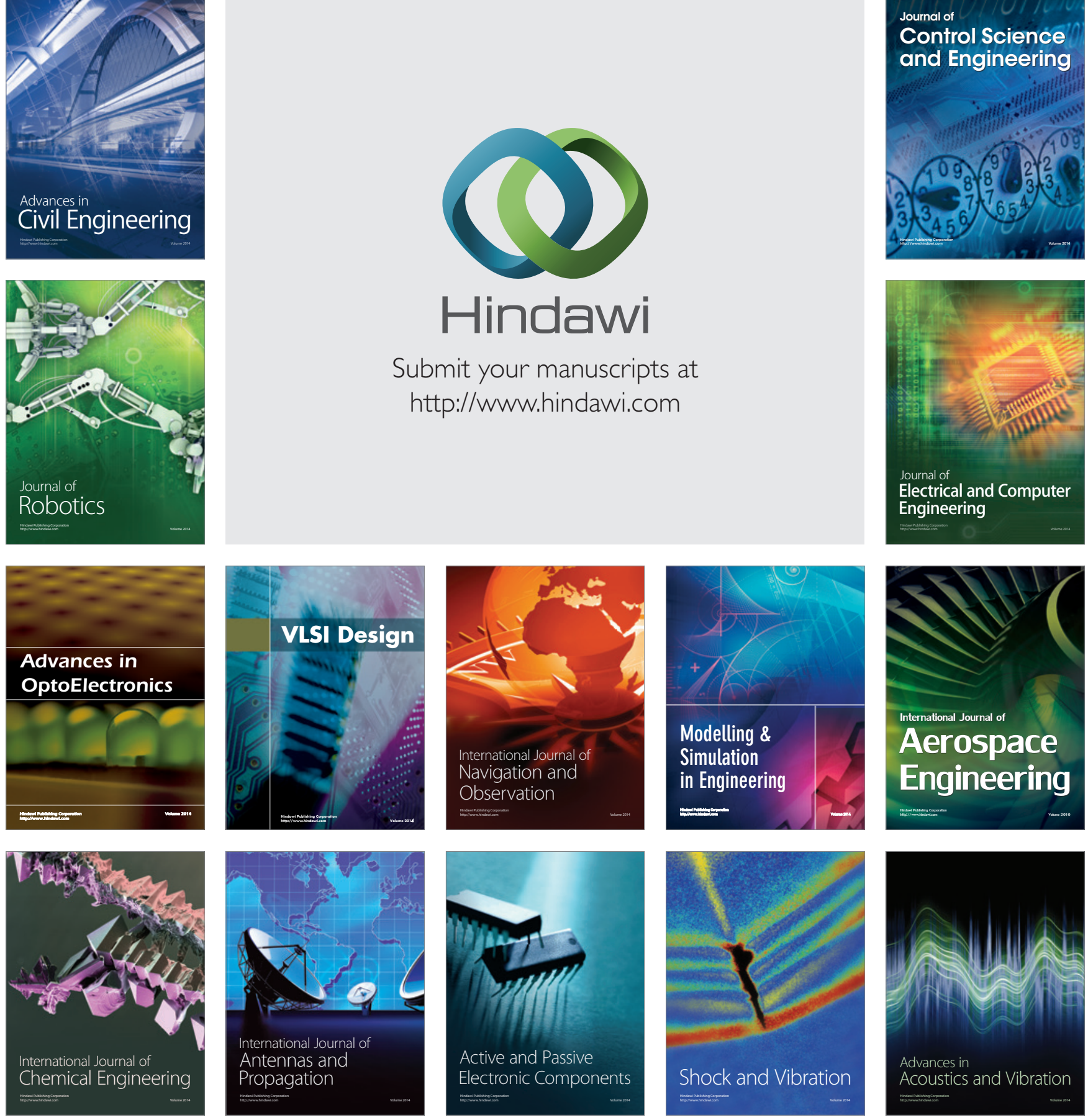